\title{
Crantor, a short-lived horseshoe companion to Uranus ${ }^{\star}$
}

\author{
C. de la Fuente Marcos and R. de la Fuente Marcos
}

\author{
Universidad Complutense de Madrid, Ciudad Universitaria, 28040 Madrid, Spain \\ e-mail: nbplanet@fis.ucm.es
}

Received 29 October 2012 / Accepted 2 January 2013

\begin{abstract}
Context. Stable co-orbital motion with Uranus is vulnerable to planetary migration, but temporary co-orbitals may exist today. So far, only two candidates have been suggested, both moving on horseshoe orbits: 83982 Crantor $\left(2002 \mathrm{GO}_{9}\right)$ and $2000 \mathrm{SN}_{331}$.

Aims. (83982) Crantor is currently classified in the group of the Centaurs by the MPC although the value of its orbital period is close to that of Uranus. Here we revisit the topic of the possible 1:1 commensurability of (83982) Crantor with Uranus, explore its dynamical past, and look into its medium-term stability and future orbital evolution.

Methods. Our analysis is based on the results of $N$-body calculations that use the most updated ephemerides and include perturbations by the eight major planets, the Moon, the barycenter of the Pluto-Charon system, and the three largest asteroids.

Results. (83982) Crantor currently moves inside Uranus' co-orbital region on a complex horseshoe orbit. The motion of this object is primarily driven by the influence of the Sun and Uranus, although Saturn plays a significant role in destabilizing its orbit. The precession of the nodes of (83982) Crantor, which is accelerated by Saturn, controls its evolution and short-term stability. Although this object follows a temporary horseshoe orbit, more stable trajectories are possible and we present $2010 \mathrm{EU}_{65}$ as a long-term horseshoe librator candidate in urgent need of follow-up observations. Available data indicate that the candidate $2000 \mathrm{SN}_{331}$ is not a Uranus' co-orbital.

Conclusions. Our calculations confirm that (83982) Crantor is currently trapped in the 1:1 commensurability with Uranus but it is unlikely to be a primordial 1:1 librator. Although this object follows a chaotic, short-lived horseshoe orbit, longer term horseshoe stability appears to be possible. We also confirm that high-order resonances with Saturn play a major role in destabilizing the orbits of Uranus co-orbitals.
\end{abstract}

Key words. methods: numerical - minor planets, asteroids: individual: Crantor (2002 GO9) - minor planets, asteroids: general minor planets, asteroids: individual: 2010 EU65 - minor planets, asteroids: individual: 2000 SN331 - celestial mechanics

\section{Introduction}

An object librating in a trajectory that encompasses the Lagrangian points $\mathrm{L}_{4}, \mathrm{~L}_{5}$ and $\mathrm{L}_{3}$ of a host planet evolves on a so-called regular horseshoe orbit. Viewed in a frame of reference that co-rotates with the host planet, the shape of such an orbit projected onto the ecliptic plane resembles that of an actual horseshoe although its three-dimensional layout looks more like a corkscrew around the orbit of the host planet while both revolve around the Sun. The size of the horseshoe orbit depends on the mass of the host planet, being wider and having a better chance of survival when this mass decreases (Dermott \& Murray 1981a). In general, horseshoe orbits are not considered to be long-term stable (Dermott \& Murray 1981a; Murray \& Dermott 1999). Horseshoe orbits were originally predicted by Brown (1911) and Darwin (1912) and further studied later by, for example, Thuring (1959), Rabe (1961), Giacaglia (1970), Weissman \& Wetherill (1974) and Garfinkel (1977), but were largely considered theoretical curiosities until the Saturnian moons Janus and Epimetheus were identified as horseshoe librators (Smith et al. 1980; Synnott et al. 1981; Dermott \& Murray 1981b). The existence of minor planets moving on horseshoe orbits around the major planets was first postulated by Milani et al. (1989) and Michel et al. (1996) on theoretical grounds.

\footnotetext{
* Figures 2 and 6 (animations) are available in electronic form at http://www . aanda.org
}

In the solar system, there are several real examples of minor bodies moving on such orbits. The first minor body to be confirmed to follow a horseshoe orbit was 3753 Cruithne (1986 TO) (Wiegert et al. 1997, 1998), in this case with the Earth. Karlsson (2004) found multiple objects moving in temporary horseshoe orbits with Jupiter. Connors et al. (2004) identified an object, $2003 \mathrm{YN}_{107}$, following a compound horseshoe-quasi-satellite orbit with the Earth. Additional objects moving in comparable trajectories are $2002 \mathrm{AA}_{29}$ and $2001 \mathrm{GO}_{2}$ (Brasser et al. 2004). $2001 \mathrm{CK}_{32}$ follows an orbit similar to that of (3753) Cruithne but hosted by Venus, not the Earth (Brasser et al. 2004). (36017) $1999 \mathrm{ND}_{43}$ is a horseshoe librator with Mars (Connors et al. 2005). Yet another horseshoe companion of the Earth was found in $2010 \mathrm{SO}_{16}$ (Christou \& Asher 2011). Additional horseshoe librators with Jupiter were recently identified by Wajer \& Królikowska (2012). Finally, (310071) $2010 \mathrm{KR}_{59}$ is following a temporary and rather complex horseshoe orbit with Neptune (de la Fuente Marcos \& de la Fuente Marcos 2012a).

Numerical simulations predict that Uranus may have retained a certain amount of its primordial co-orbital minor planet population (Holman \& Wisdom 1993; Wiegert et al. 2000; Nesvorný \& Dones 2002; Marzari et al. 2003). However, Uranus appears not to be able to efficiently capture objects into the 1:1 commensurability today even for short periods of time (Horner \& Evans 2006). The stability of hypothetical Uranus co-orbitals, specifically those moving in tadpole orbits, has been studied by Dvorak et al. (2010), who have found that the orbital 
inclination is the key parameter regarding stability, only the inclination intervals $(0,7)^{\circ},(9,13)^{\circ},(31,36)^{\circ}$ and $(38,50)^{\circ}$ appear to be stable. This scarcity of Uranus co-orbitals seems to be confirmed by current observational results. Although hundreds of objects have been discovered in the outer solar system during the various wide-field surveys carried out during the past decade, the two objects pointed out by Gallardo (2006) remain as the only Uranus' co-orbital candidates identified to date.

Calculations by Gallardo (2006) revealed that two objects were moving in a 1:1 mean motion resonance with Uranus. One of them, 83982 Crantor $\left(2002 \mathrm{GO}_{9}\right)$, is the main object of study of this paper. Since 2006, the orbit of this object has been improved and here we make use of the most updated ephemerides to reassess the current dynamical status of this minor body. In this paper, we use $N$-body simulations to confirm the co-orbital nature with Uranus of the asteroid (83982) Crantor, currently classified as Centaur by both the Minor Planet Center (MPC) and the Jet Propulsion Laboratory (JPL). The numerical model is described in the next section and available data on (83982) Crantor are presented in Sect. 3. The results of our $N$-body calculations are shown in Sect. 4. These results are discussed in Sect. 5. A new Uranus' horseshoe librator candidate is presented in Sect. 6 and our conclusions are summarized in Sect. 7.

\section{Numerical integration}

The orbital evolution of 83982 Crantor $\left(2002 \mathrm{GO}_{9}\right)$ was computed for 0.5 Myr forward and backward in time using the Hermite integration scheme described by Makino (1991) and implemented by Aarseth (2003). This $N$-body code has been extensively tested by the authors and used in a variety of recent solar system numerical studies (de la Fuente Marcos \& de la Fuente Marcos 2012a,b,c,d). The standard version of this sequential code is publicly available from the IoA web site ${ }^{1}$. Our integrations include the perturbations by the eight major planets, the Moon, the barycenter of the Pluto-Charon system, and the three largest asteroids. For accurate initial positions and velocities we used the heliocentric ecliptic Keplerian elements provided by the Jet Propulsion Laboratory on-line solar system data service $^{2}$ (Giorgini et al. 1996) and initial positions and velocities based on the DE405 planetary orbital ephemerides (Standish 1998) referred to the barycenter of the solar system. In addition to the orbital calculations completed using the nominal elements in Table 1, we have performed 50 control simulations with sets of orbital elements obtained from the nominal ones and the quoted uncertainties $(3-\sigma)$. The derived sample of control orbits follows a Gaussian distribution in the six-dimensional space of orbital elements and they are compatible with the observations within the 3- $\sigma$ uncertainties. The analysis of the control orbits provides some insight into the predictability of the trajectory of the object. The numerical rather than analytical approach to the study of this object is more appropriate because planets other than Uranus, as we will discuss later, play a role on the dynamics of this asteroid, rendering the traditional perturbational approach within the framework of the three-body problem particularly limited in this case. There exists practically no analytical means to study horseshoe orbits.

\footnotetext{
1 http://www.ast.cam.ac.uk/ sverre/web/pages/nbody.htm 2 http://ssd.jpl.nasa.gov/?planet_pos
}

Table 1. Heliocentric Keplerian orbital elements of 83982 Crantor (2002 $\mathrm{GO}_{9}$ ) used in this research.

\begin{tabular}{llc}
\hline \hline Semi-major axis, $a$ & $=$ & $19.3553 \pm 0.0014 \mathrm{AU}$ \\
Eccentricity, $e$ & $=$ & $0.27496 \pm 0.00004$ \\
Inclination, $i$ & $=$ & $12.78489 \pm 0.00003^{\circ}$ \\
Longitude of the ascending node, $\Omega$ & $=$ & $117.4097 \pm 0.0003^{\circ}$ \\
Argument of perihelion, $\omega$ & $=$ & $92.599 \pm 0.003^{\circ}$ \\
Mean anomaly, $M$ & $=$ & $43.964 \pm 0.006^{\circ}$ \\
Perihelion, $q$ & $=$ & $14.0333 \pm 0.0002 \mathrm{AU}$ \\
Aphelion, $Q$ & $=$ & $24.677 \pm 0.002 \mathrm{AU}$ \\
Absolute magnitude, $H$ & $=$ & $8.5 \pm 0.8 \mathrm{mag}$ \\
\hline
\end{tabular}

Notes. Values include the $1-\sigma$ uncertainty. The orbit is based on $104 \mathrm{ob}-$ servations spanning a data-arc of 2,654 days or $7.27 \mathrm{yr}$, from 2001-0320 to 2008-06-25. (Epoch = JD 2456 200.5, 2012-Sep.-30.0; J2000.0 ecliptic and equinox. Source: JPL Small-Body Database.)

\section{83982 Crantor $\left(2002 \mathrm{GO}_{9}\right)$ in perspective}

83982 Crantor (2002 $\mathrm{GO}_{9}$ ) was discovered on April 12, 2002 by Helin, Pravdo, Lawrence, Hicks and Thicksten working for the Near-Earth Asteroid Tracking (NEAT) project at Palomar Observatory (Gilmore et al. 2002). It was originally reported as a scattered-disk object with $a=54.24$ AU and $e=0.81$ but soon after, a number of precovery images of the object were uncovered: it first appears in images obtained on March 20, 2001 from the Air Force Maui Optical and Supercomputing (AMOS) observatory located at the summit of Haleakala, then on images acquired on April 16, 2001 from the Apache Point Observatory as part of the Sloan Digital Sky Survey (SDSS), and again on new images obtained on March 26, 2001 and January 15, 2002 from Haleakala-AMOS (Ticha et al. 2002). All this observational material enabled the computation of a reliable orbit characterized by a value of the semi-major axis (19.36 AU) close to that of Uranus, significant eccentricity $(\sim 0.3)$, and moderate inclination $\left(\sim 13^{\circ}\right)$. Therefore, its orbit is now relatively well determined with 104 observations spanning a data-arc of 2654 days and it is clearly not compatible with that of a scattered-disk object. Consistently, (83982) Crantor is currently listed by both the Minor Planet Center (MPC) Database ${ }^{3}$ and the JPL Small-Body Database $^{4}$ as a Centaur.

Early photometric work (Tegler et al. 2003) pointed out the red surface color of (83982) Crantor and suggested that this object and many others like it were formed farther away from the Sun than distant minor bodies characterized by gray surface color. In fact, (83982) Crantor is one of the reddest objects of the solar system, close to fellow ultra-red Centaur (5145) Pholus; its surface should be partially covered by tholins to explain its redness and low albedo (Cruikshank et al. 2007). The rotation period of (83982) Crantor is $6.97 \mathrm{~h}$ or $9.67 \mathrm{~h}$ with a light-curve amplitude of 0.14 mag (Ortiz et al. 2003). (83982) Crantor was observed with the near-infrared integral field spectrograph SINFONI at the Very Large Telescope (VLT) that found evidence of an absorption feature in its spectrum at $2.0 \mu \mathrm{m}$, probably associated with water ice and another feature at $2.3 \mu \mathrm{m}$, which could be associated with methanol (Alvarez-Candal et al. 2007). These results confirmed previous hints obtained by Doressoundiram et al. (2005). Visible spectra further support the very red nature of the object (Alvarez-Candal et al. 2008). Additional near-infrared spectra taken with the Keck I Telescope confirmed previous

\footnotetext{
3 http://www.minorplanetcenter.net/db_search

4 http://ssd.jpl.nasa.gov/sbdb.cgi
} 
results (Barkume et al. 2008). Near-infrared photometry was obtained by Doressoundiram et al. (2007). Incomplete photometry was obtained with FORS1 at VLT (DeMeo et al. 2009). Recent Hubble Wide Field Camera 3 results (Fraser \& Brown 2012) validate previous findings, indicating the presence of irradiated organics and tholins on its surface.

The asteroid (83982) Crantor is relatively large. The object has a diameter of $<66.7_{-19.6}^{+18.7} \mathrm{~km}$ with visible geometric albedo of $8.60_{-3.36}^{+8.62} \%$ (Stansberry et al. 2008). Its period of revolution around the Sun, approximately $85.15 \mathrm{yr}$ at present, is very close to that of Uranus, $84.32 \mathrm{yr}$. As a result, (83982) Crantor and Uranus appear to follow each other in their paths around the Sun, although (83982) Crantor's orbital plane is currently tilted to that of the Earth by $12.8^{\circ}$ (Uranus' is $0.8^{\circ}$ ). Its dynamical half-lives have been estimated to be $2.93 \mathrm{Myr}$ (for forward integration) and 3.67 Myr (for backward integration) by Horner et al. (2004). (83982) Crantor was originally proposed as a possible co-orbital of Uranus together with $2000 \mathrm{SN}_{331}$ by Gallardo (2006). Both objects would be following horseshoe trajectories. In the same research work, it is pointed out that Uranus' Trojans are affected by high-order resonances with Saturn. The asteroid $2000 \mathrm{SN}_{331}$ has not been reobserved since its discovery and its orbit remains very poorly known (see below). In the following section we focus on the dynamical evolution of (83982) Crantor.

\section{Dynamical evolution}

In order to study the librational properties of 83982 Crantor (2002 $\mathrm{GO}_{9}$ ) and following the work of Mikkola et al. (2006), we define the relative deviation of the semi-major axis from that of Uranus by $\alpha=\left(a-a_{\mathrm{U}}\right) / a_{\mathrm{U}}$, where $a$ and $a_{\mathrm{U}}$ are the semimajor axes of the object and Uranus, respectively, and also the relative mean longitude $\lambda_{\mathrm{r}}=\lambda-\lambda_{\mathrm{U}}$, where $\lambda$ and $\lambda_{\mathrm{U}}$ are the mean longitudes of the object and Uranus, respectively. If $\lambda_{\mathrm{r}}$ oscillates around $0^{\circ}$, the object is considered a quasi-satellite; Trojan bodies are characterized by $\lambda_{\mathrm{r}}$ oscillating around $+60^{\circ}\left(\mathrm{L}_{4}\right.$ Trojan) or $-60^{\circ}$ (or $300^{\circ}, \mathrm{L}_{5}$ Trojan); finally, an object librating with amplitude $>180^{\circ}$ follows a horseshoe orbit (see, e.g., Murray \& Dermott 1999).

Our $N$-body calculations confirm that (83982) Crantor currently is a co-orbital companion to Uranus and follows a horseshoe orbit, all in agreement with what was originally pointed out by Gallardo (2006), see Fig. 1. The apparent overlap with Uranus' position in Fig. 1 is the result of the moderate orbital inclination of the object. The orbital behavior of (83982) Crantor is illustrated by the animation displayed in Fig. 2 (available on the electronic edition as a high resolution animation or embedded at lower resolution in the pdf file associated to this paper). The orbit is presented in three frames of reference: heliocentric (left), co-rotating with Uranus (top-right) and Uranocentric (bottom-right). (83982) Crantor moves in a non-regular horseshoe orbit with a period of about 8500 years. Non-regular means that compound horseshoe-quasi-satellite loops are possible. The dynamical evolution of an object moving in a horseshoe orbit associated to Uranus can be decomposed into a slow guiding center motion and a superimposed short period three-dimensional epicyclic motion viewed in a frame of reference co-rotating with Uranus. The object spirals along Uranus' orbit at a rate of nearly 0.08 per year, but each time it gets close to Uranus, it is effectively repelled by the planet. The reversals of the net motion of the object with respect to Uranus are obvious in the accompanying animation and because they take place when the distance to Uranus is the smallest, the gravitational interaction with

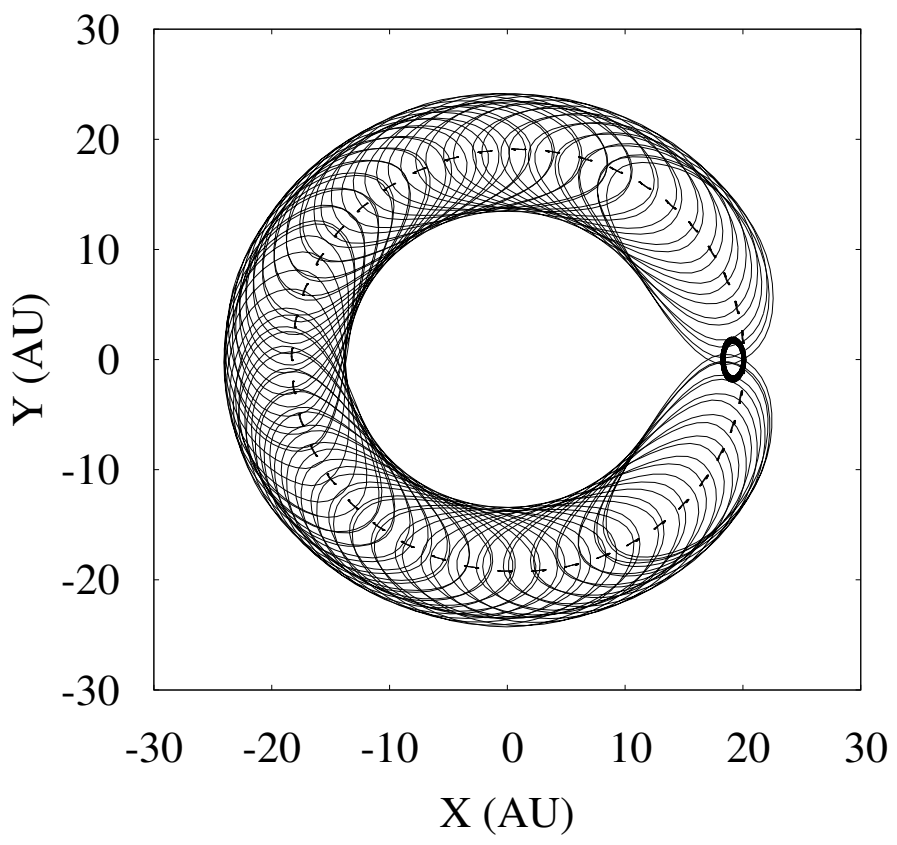

Fig. 1. Motion of 83982 Crantor $\left(2002 \mathrm{GO}_{9}\right)$ over the time range (-3, 5) kyr is displayed projected onto the ecliptic plane in a coordinate system rotating with Uranus. The orbit and the position of Uranus are also indicated. In this frame of reference and as a result of its non-negligible eccentricity, Uranus describes a small ellipse. The trajectory of the object spirals along Uranus' orbit at a rate of nearly 0.08 per year.

Uranus is, at that moment, the strongest. Although the inclination of the asteroid is high enough to avoid close encounters with Uranus when the relative mean longitude approaches zero, these close encounters that can only occur in the vicinity of the nodes play a major role on the activation and deactivation of the horseshoe behavior of this object (see below).

The relative deviation of the semi-major axis, $\alpha$, as a function of the relative mean longitude, $\lambda_{\mathrm{r}}$, is displayed in Fig. 3, for selected time intervals. The relative mean longitude of (83982) Crantor librates around the unstable Lagrangian point $\mathrm{L}_{3}$ at $180^{\circ}$ with large amplitude, allowing the object to come quite close to Uranus and to suffer destabilizing close encounters. Typical horseshoe behavior is observed, but brief (half a loop) quasisatellite episodes also take place. During these events, the object still moves in a 1:1 commensurability with Uranus but $\lambda_{\mathrm{r}}$ librates about $0^{\circ}$. This exchange between horseshoe and quasi-satellite paths (or compound horseshoe-quasi-satellite orbits) is observed in other horseshoe librators, for example 3753 Cruithne (1986 TO) (Wiegert et al. 1997, 1998).

A plot of the orbital elements of (83982) Crantor over a $100 \mathrm{kyr}$ interval centered on the present is shown in Fig. 4. The distance of (83982) Crantor from Uranus displayed in Fig. 4, panel A shows that the object undergoes close encounters with Uranus. A very close encounter, almost a collision at $0.17 \mathrm{AU}$ or 0.37 Hill radii (which is $0.45 \mathrm{AU}$ for Uranus), took place $19248 \mathrm{yr}$ ago. The evolution of $\lambda_{\mathrm{r}}$ in panel $\mathrm{B}$ indicates that the current horseshoe episode will end in about $20000 \mathrm{yr}$ from now; (83982) Crantor will decouple from Uranus with $\lambda_{\mathrm{r}}$ circulating, not librating. The semi-major axis exhibits an oscillatory behavior that is characteristic of the effects of a $1: 1$ mean motion resonance. The eccentricity decreases by $10 \%$ during the very close encounter pointed out above and then increases again after nearly 19000 yr, see Fig. 4, panel D. During the horseshoe episode, the orbital inclination remains in the interval $(12,13)^{\circ}$, 

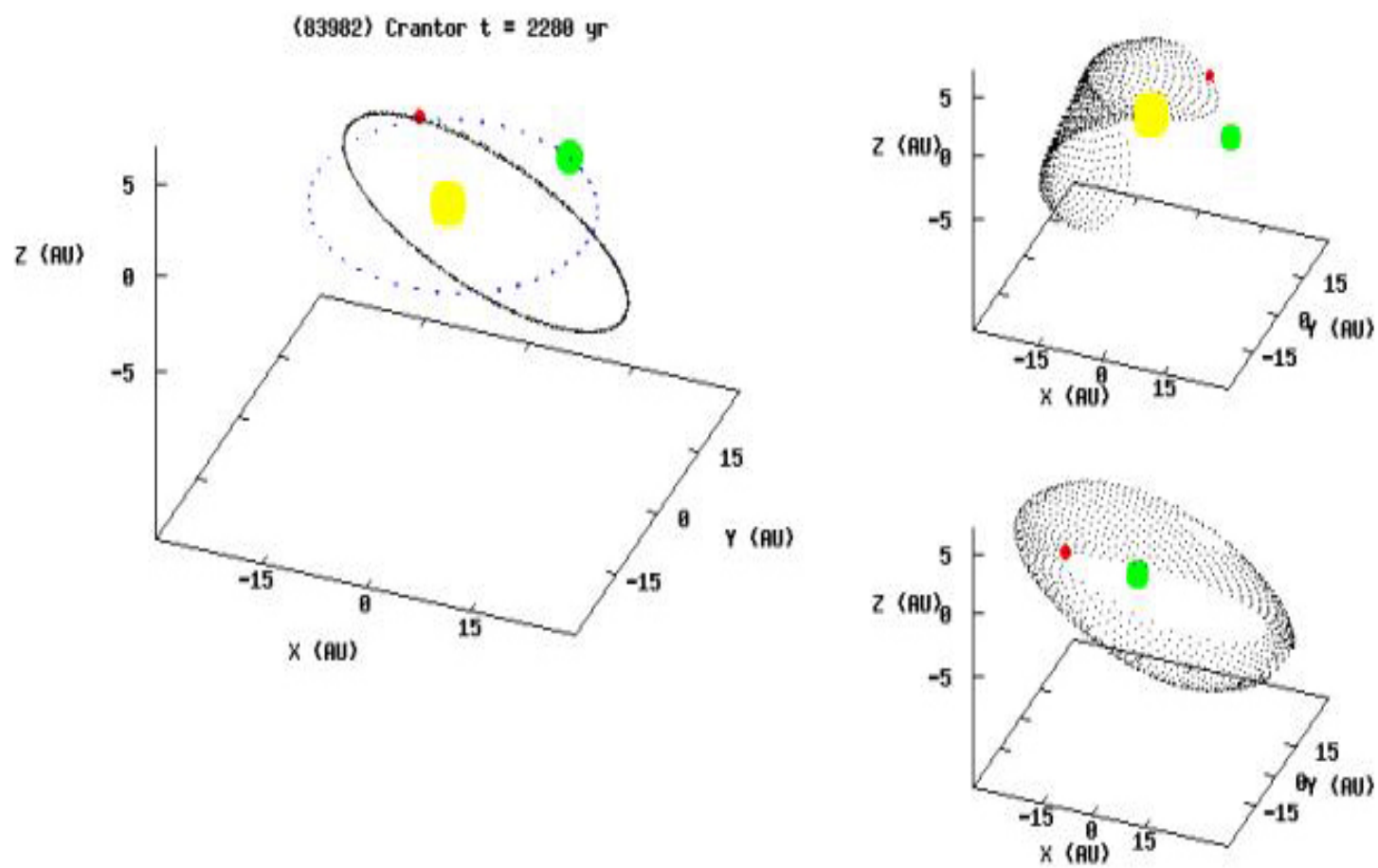

Play/Pause

Fig. 2. Three-dimensional evolution of the orbit of 83982 Crantor $\left(2002 \mathrm{GO}_{9}\right)$ in three different frames of reference: heliocentric (left), frame co-rotating with Uranus but centered on the Sun (top-right), and uranocentric (bottom-right). The red point is (83982) Crantor, the green one is Uranus, and the yellow one is the Sun. The osculating orbits are outlined and the viewing angle changes slowly to facilitate visualizing the orbital evolution. Still from the animated figure available online only.

see Fig. 4, panel E. The argument of perihelion, Fig. 4, panel F, circulates. In Fig. 4, panel B, we also show the evolution of the relative mean longitude for a particular control orbit that has been chosen close to the 3- $\sigma$ limit; in this way, its orbital elements are most different from the nominal ones in Table 1. For this particular orbit and prior to entering the horseshoe dynamical state, the object was an $\mathrm{L}_{4}$ Trojan. All control calculations indicate that (83982) Crantor has been co-orbital with Uranus for at least $100 \mathrm{kyr}$.

\section{Discussion}

The characteristic e-folding time of 83982 Crantor (2002 $\mathrm{GO}_{9}$ ) during the present horseshoe dynamical state has been found to be about $1 \mathrm{kyr}$. This value gives the reader the idea of how small the timescale required for two initially infinitesimally close trajectories is in this case to separate significantly. An additional test that confirms how sensitive to small changes the dynamical evolution of (83982) Crantor is, can be obtained by repeating the calculations, this time excluding one of the three largest asteroids; the orbit significantly diverges from the standard one after just a few 10 kyr. Therefore, simulations over long timescales (e.g. 1 Myr) are not appropriate in this case and therefore we restrict our figures to a few $10 \mathrm{kyr}$. Since the orbit of the asteroid is chaotic, its true phase-space trajectory will diverge exponentially from that obtained in our calculations. However, the evolution of the control orbits exhibits a very similar secular behavior of the orbital elements in the time interval $(-10,10)$ kyr. We also note that at the ends of the interval, a close encounter between the asteroid and Uranus happened. Therefore, the dynamical evolution of (83982) Crantor as described by our integrations can be considered to be reliable within that relatively short time interval, but beyond that we regard our results as an indication of the probable dynamical behavior of the object.

Our calculations show that the orbit of (83982) Crantor is predictable only within a relatively short time interval. We consider that an orbit is predictable when all control orbits give comparable results. Clearly, the current horseshoe behavior is not stable on a timescale longer than a few $10 \mathrm{kyr}$. The analysis of the control orbits indicates that this object may have been coorbital for less than $200 \mathrm{kyr}$ and it may leave Uranus' co-orbital region in less than 100 kyr. However, some control orbits remain in the co-orbital region for about $1 \mathrm{Myr}$. About half the studied control orbits started co-orbital motion about 10-20 kyr ago but one third left the circulation regime to become coorbitals about $80 \mathrm{kyr}$ ago. The rest appear to have been switching between the various co-orbital states for several $100 \mathrm{kyr}$. The changes in dynamical state are always associated to close encounters with Uranus. Regarding the future orbital evolution of (83982) Crantor, the majority $(70 \%)$ of control orbits continue in the horseshoe state for about $15 \mathrm{kyr}$. The remaining orbits divide evenly among those lasting less and those lasting more than 15 kyr. Twenty per cent of the control orbits leave the coorbital region permanently after ending their horseshoe stage but 


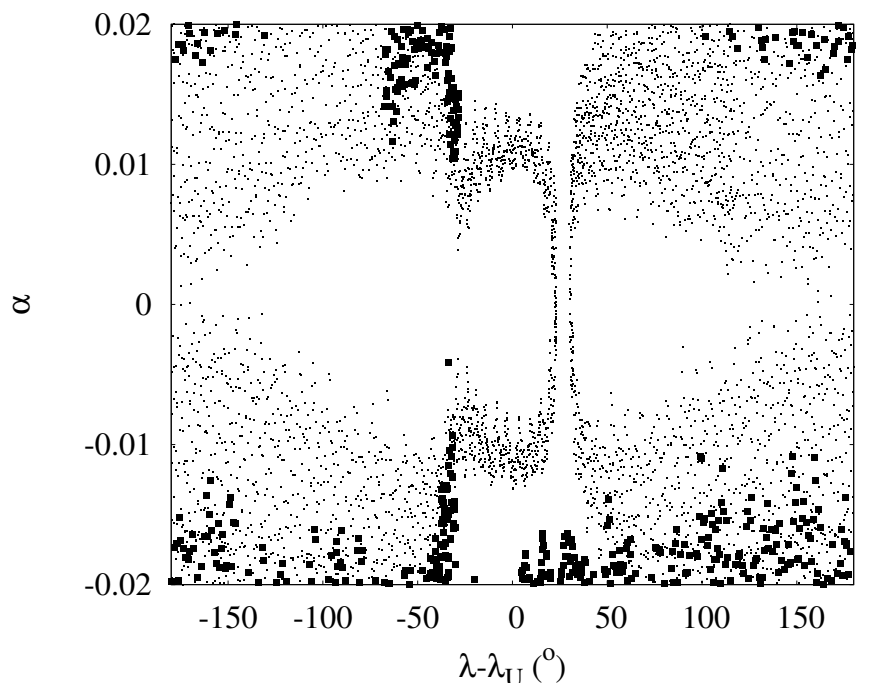

Fig. 3. Resonant evolution of the asteroid 83982 Crantor (2002 $\left.\mathrm{GO}_{9}\right)$. The relative deviation of its semi-major axis from that of Uranus, $\alpha$, as a function of the relative mean longitude, $\lambda_{\mathrm{r}}$, during the time intervals $(-22,-10) \mathrm{kyr}$ (black squares) and $(2,12) \mathrm{kyr}$ (dots) is displayed. The first interval covers the close encounter observed in Fig. 4, panel A (see the text). The second interval lasts an entire cycle of the horseshoe orbit and includes a quasi-satellite half libration, i.e., $\lambda_{\mathrm{r}}$ goes beyond $-60^{\circ}$ passes $0^{\circ}$ but does not reach $60^{\circ}$.

nearly $70 \%$ return after 5 to $15 \mathrm{kyr}$. The rest switch to the quasisatellite phase. Out of the returning co-orbitals, the vast majority make a comeback as horseshoe librators with only $15 \%$ becoming quasi-satellites or Trojans. Nearly $25 \%$ continue switching between the various co-orbital states for several $100 \mathrm{kyr}$ and the rest leave the co-orbital region after nearly $80 \mathrm{kyr}$. Among the coorbital states, the vast majority of the episodes produce irregular horseshoe orbits and they also last longer; quasi-satellite events are a distant second and tadpole orbits are observed in just a few cases. Most discrete episodes last for less than 10 kyr. Clearly, it is quite difficult to provide a clean picture of the medium-term past and future of this object. The amount of time spent in the horseshoe state prior to the origin of time considered in this research is similar in all the control models and in most of them the relative mean longitude circulates before entering and after leaving the state, but the details beyond $20 \mathrm{kyr}$ into the past and after $30 \mathrm{kyr}$ into the future are quite heterogeneous both in terms of the actual type of orbital behavior and its duration.

The asteroid 83982 Crantor $\left(2002 \mathrm{GO}_{9}\right)$ follows an eccentric orbit ( $e \approx 0.3$ ) but currently, it only crosses the orbit of Uranus. In general, minor bodies that cross the paths of one or more planets can be rapidly destabilized by scattering resulting from close planetary approaches if their orbital inclinations are low. (83982) Crantor moves in a relatively highly inclined orbit $\left(i \approx 13^{\circ}\right)$. In the solar system and for a minor body moving in an inclined orbit, close encounters with major planets are only possible in the vicinity of the nodes. The distance between the Sun and the nodes is given by $r=a\left(1-e^{2}\right) /(1 \pm e \cos \omega)$, where the + sign denotes the ascending node and the - sign the descending node. Figure 5 shows the evolution of the distance to the nodes of (83982) Crantor in the time range $(-50,50) \mathrm{kyr}$. The evolution of the orbital elements in Fig. 4 shows that changes in $\omega$ dominate those in $a$ and $e$ for (83982) Crantor and largely control the positions of the nodes. The current precession rate of the nodes is nearly +0.2 per century. This value decreases when $\lambda_{\mathrm{r}}$ circulates. We found that, in accordance with theory (Namouni 1999), if (83982) Crantor moves in a horseshoe orbit, $\dot{\omega}>0$ but during the

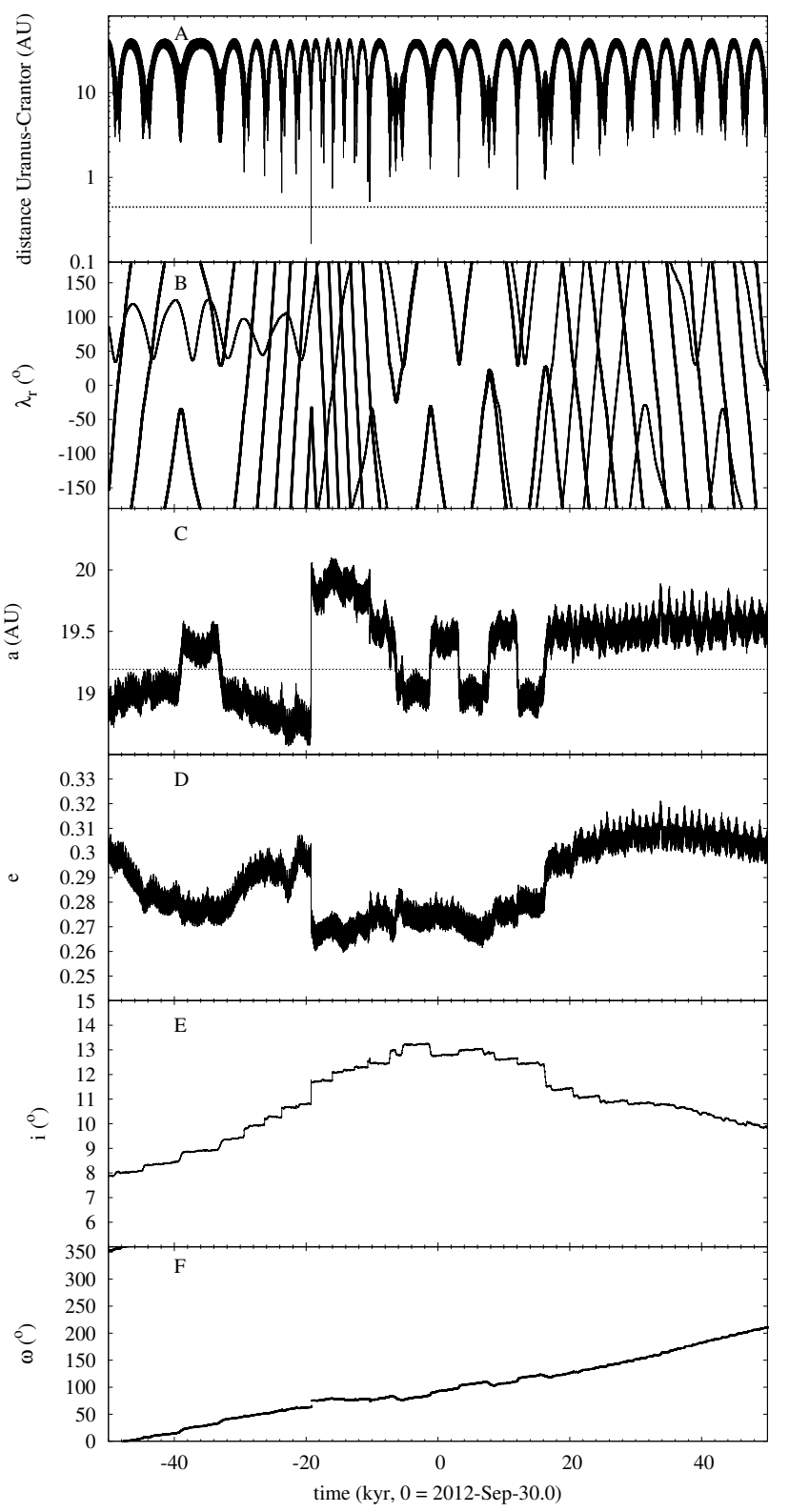

Fig. 4. Time evolution of various parameters. The distance of 83982 Crantor $\left(2002 \mathrm{GO}_{9}\right.$ ) from Uranus (panel A)); the value of the Hill sphere radius of Uranus, $0.447 \mathrm{AU}$, is displayed. The resonant angle, $\lambda_{\mathrm{r}}($ panel B)) for the nominal orbit in Table 1 (thick line) and one of the control orbits (thin line). This particular control orbit was chosen close to the 3- $\sigma$ limit so its orbital elements are most different from the nominal ones. The orbital elements $a$ (panel C)) with the current value of Uranus' semi-major axis, $e(\operatorname{panel} \mathbf{D})), i(\operatorname{panel} \mathbf{E}))$, and $\omega($ panel $\mathbf{F}))$.

brief quasi-satellite loops, the value of the argument of perihelion decreases (see Fig. 4, panel F). The values of the nodal distances are currently very close to the value of the semi-major axis of Uranus. Close encounters are therefore possible at both nodes, doubling the probability of having a strong gravitational interaction with Uranus that may significantly change the orbit. When the object is east of Uranus, encounters occur at the ascending node. In contrast, close encounters take place at the descending node when (83982) Crantor approaches Uranus from the west. In this way, the gravitational perturbations from Uranus are most effective, and both Saturn and Neptune are secondary perturbers for this object. Currently and as a result of its horseshoe trajectory, the object approaches Uranus every 4354 yr although most of the time the asteroid remains at a safe distance from Uranus. 


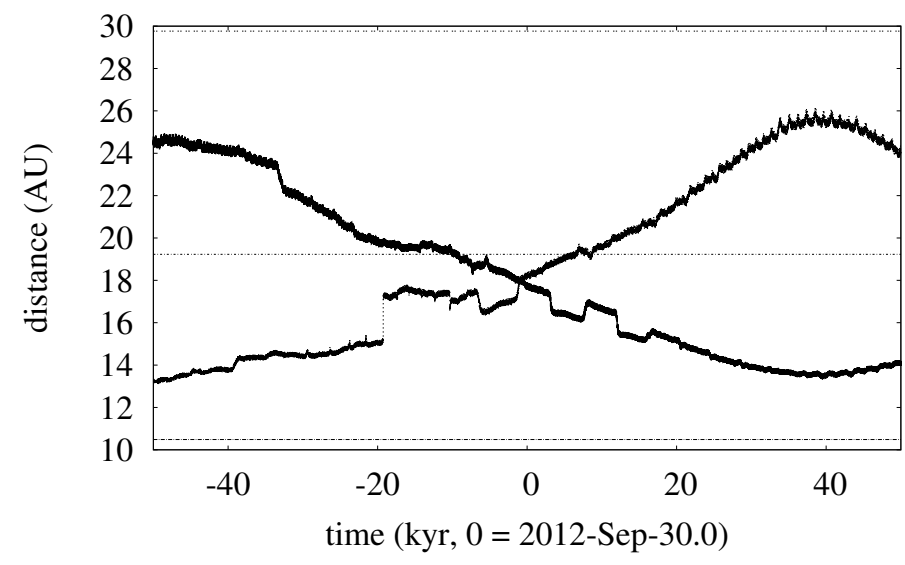

Fig. 5. Heliocentric distance to the descending (thick line) and ascending nodes (dotted line) of 83982 Crantor $\left(2002 \mathrm{GO}_{9}\right)$. Saturn's aphelion, Uranus' semi-major axis, and Neptune's perihelion distances are also shown. Both nodal distances are at present relatively close to the value of Uranus' semi-major axis, which explains why close encounters with Uranus are possible and relatively frequent, but most of the time the asteroid remains at a safe distance from Uranus.

Although (83982) Crantor's eccentricity is not high enough to cross Saturn's orbit, Saturn appears to play a non-negligible role in destabilizing the object's motion. Even if close encounters with Saturn and Neptune are not possible, this object (together with Uranus) currently moves in near resonance with the other three giant planets: 1:7 with Jupiter, 7:20 with Saturn, and 1:2 with Neptune. To study the role of these three giant planets on the resonant evolution of (83982) Crantor, we repeated the calculations considering negligible masses for Jupiter, Saturn and Neptune. This alteration removes the associated near resonance. The "toy model" without Neptune gives very similar results for the evolution of this minor body. Therefore, the role of the near resonance with Neptune can be regarded as negligible. In sharp contrast, the cases of the toy models without Jupiter or Saturn are dramatically different. The lack of Jupiter has immediate effects on the results because it amplifies the dynamical effect of Saturn, but the absence of Saturn has major effects on the overall orbital evolution of (83982) Crantor. The precession rate decreases by $30 \%$ and the object remains as a complex Uranus horseshoe librator for several 100 kyr. Though (83982) Crantor moves primarily under the influence of the Sun and Uranus, Saturn (mainly), Jupiter and Neptune play an important role by influencing, through torque-induced precession, the position of the asteroid's nodes. Variations in the nodal distance strongly affect the interaction of (83982) Crantor with Uranus and may change or terminate the horseshoe orbit currently observed. This precession of the nodes is the mechanism by which minor planets are placed in or are removed from horseshoe orbits (Wiegert et al. 1998). On the other hand, the repetitive episodes described above (see also Fig. 4, panel B) in which (83982) Crantor's relative mean longitude librates for several cycles, then circulates for a few more cycles before restarting libration once again are characteristic of a type of dynamical behavior known as resonance angle nodding, see Ketchum et al. (2013). These authors concluded that nodding often occurs when a small body is in an external (near) mean motion resonance with a larger planet. This type of complicated dynamics has been observed in other horseshoe librators.

Gallardo (2006) suggested that two objects were trapped in a 1:1 mean motion resonance with Uranus, both moving on horseshoe orbits: (83982) Crantor and $2000 \mathrm{SN}_{331}$. We have just
Table 2. Heliocentric Keplerian orbital elements of $2010 \mathrm{EU}_{65}$ used in this research.

\begin{tabular}{llc}
\hline \hline Semi-major axis, $a$ & $=$ & $19.2041 \mathrm{AU}$ \\
Eccentricity, $e$ & $=$ & 0.05402 \\
Inclination, $i$ & $=$ & $14.8382^{\circ}$ \\
Longitude of the ascending node, $\Omega$ & $=$ & $4.61965^{\circ}$ \\
Argument of perihelion, $\omega$ & $=$ & $180.824^{\circ}$ \\
Mean anomaly, $M$ & $=$ & $0.37496^{\circ}$ \\
Perihelion, $q$ & $=$ & $18.1668 \mathrm{AU}$ \\
Aphelion, $Q$ & $=$ & $20.2414 \mathrm{AU}$ \\
Absolute magnitude, $H$ & $=$ & $9.1 \mathrm{mag}$ \\
\hline
\end{tabular}

Notes. The orbit is based on 26 observations spanning a data-arc of 85 days, from 2010-03-13 to 2010-06-06. $($ Epoch = JD2 455 300.5, 2010-Apr-14.0; J2000.0 ecliptic and equinox. Source: JPL Small-Body Database.)

confirmed that (83982) Crantor actually moves in a relatively short-lived horseshoe orbit associated to Uranus. However, we cannot do the same with the other object, $2000 \mathrm{SN}_{331}$. Our calculations indicate that this object is not co-orbital but may move in a temporary 10:9 inner resonance with Uranus, i.e., the object completes ten revolutions around the Sun while Uranus moves around the Sun just nine times. This result is robust, in principle, but the reliability of the orbit of this object is extremely poor because it is based on just six observations with a data-arc span of only one day. Therefore, we may say that it is a candidate to move in resonance with Uranus, but not a co-orbital.

Given the significant destabilizing role played by Saturn on the orbital evolution of (83982) Crantor, the question concerning the existence of long-term Uranus co-orbitals moving on stable orbits takes a new twist: are they at all possible in the light of our present results? In the following section, we present early results on an object that, because it moves in a low-eccentricity orbit, may be able to survive as Uranus co-orbital for a longer period of time. In any case, it must be pointed out that longer calculations (several Myr long) suggest that (83982) Crantor may become (600 kyr from now) a long-term Uranus' $\mathrm{L}_{5}$ Trojan. However, and given the chaotic nature of the orbit of this object, this result should be taken with caution.

\section{6. $2010 \mathrm{EU}_{65}$ : a promising Uranus horseshoe candidate}

Asteroid 2010 EU $_{65}$ was discovered on March 13, 2010 in images obtained by the European Southern Observatory (ESO) at La Silla (Rabinowitz et al. 2012). The object was reobserved in the following days from the same location and also from Cerro Tololo Observatory at La Serena (Rabinowitz et al. 2010). In total, there are 26 observations with a data-arc span of 85 days. At the time of discovery, its apparent magnitude in $R$ was estimated to be 21.2. The heliocentric Keplerian orbital elements of 2010 EU $_{65}$ appear in Table 2. As a relatively recent discovery, its orbit is poorly constrained and it is included here mainly to encourage follow-up observations. Little is known of the physical properties of this object, with the exception of its absolute magnitude of 9.1. This probably suggests a medium-sized object with an estimated diameter in the range $28-90 \mathrm{~km}$, for an albedo range of $0.5-0.05$. Its period, $84.16 \mathrm{yr}$, matches that of Uranus well, $84.32 \mathrm{yr}$, so it appears to follow a 1:1 resonant orbit with Uranus, yet it is classified as a Centaur by the MPC. In general and with the exception of the inclination, its orbit is remarkably similar to that of Uranus. Our calculations for the nominal orbit 

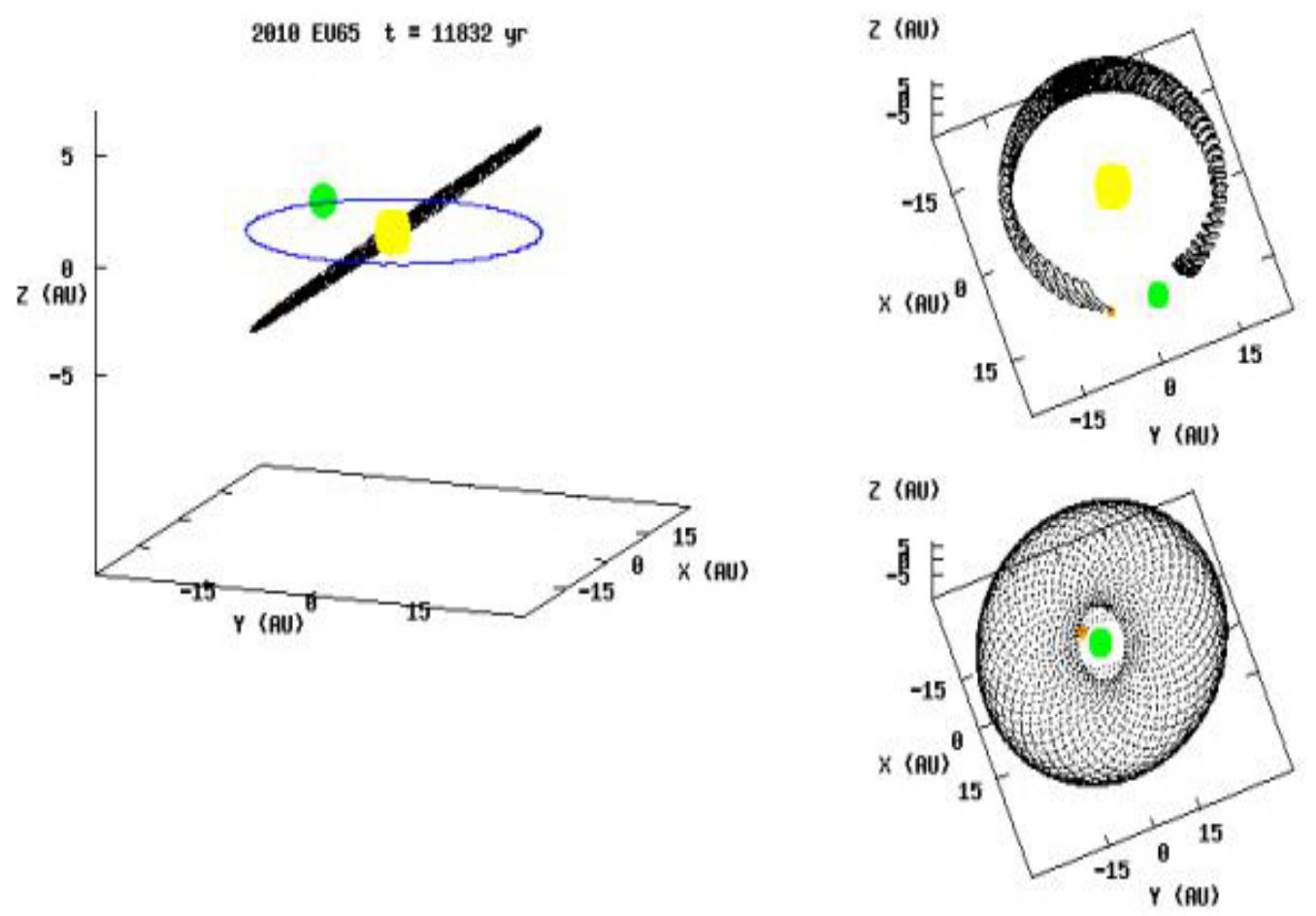

Play/Pause

Fig. 6. Three-dimensional evolution of the orbit of $2010 \mathrm{EU}_{65}$ in three different frames of reference: heliocentric (left), frame co-rotating with Uranus but centered on the Sun (top-right), and uranocentric (bottom-right). The orange point represents $2010 \mathrm{EU}_{65}$, the green one is Uranus, and the yellow one is the Sun. The osculating orbits are outlined and the viewing angle changes slowly to facilitate visualizing the orbital evolution. Still from the animated figure available online only.

in Table 2 indicate that $2010 \mathrm{EU}_{65}$ also follows a horseshoe orbit, this time very regular, associated to Uranus. In this case and due to its small eccentricity, the orbit is far more stable than that of 83982 Crantor $\left(2002 \mathrm{GO}_{9}\right)$; now the effect of Saturn is fairly negligible and the object always remains at a safe distance from Uranus. The immediate future orbital evolution of $2010 \mathrm{EU}_{65}$ is illustrated by the animation displayed in Fig. 6 (available in the electronic edition as a high-resolution animation or embedded at lower resolution in the pdf file associated to this paper). As in the case of (83982) Crantor, the orbit is presented in three frames of reference: heliocentric (left), co-rotating with Uranus (top-right), and uranocentric (bottom-right). Our numerical integrations suggest that, in sharp contrast with (83982) Crantor, this object remains in co-orbital motion with Uranus for Myr timescales. Due to its poorly known orbit, we must insist that the object is a mere horseshoe librator candidate (and in dire need of follow-up observations) although all studied control orbits (with errors below $1 \%)$ give consistent results.

\section{Conclusions}

We have analyzed the orbital behavior of Uranus' current horseshoe librator 83982 Crantor $\left(2002 \mathrm{GO}_{9}\right)$ numerically to better understand its current dynamical status, past dynamics, and future evolution as well as to gain some insight into its stability. We also showed that the evolution of this object is mainly controlled by the Sun and Uranus. In fact, close encounters with Uranus generate instability in its orbit and throw the object into and out of the horseshoe dynamical state.

(83982) Crantor is remarkable in several respects: it is the first known minor body to be trapped in a 1:1 mean motion resonance with Uranus; it currently moves in a complex, horseshoelike orbit when viewed in a frame of reference co-rotating with Uranus; and it could be the "Rosetta Stone" for understanding why the overall number of Uranus co-orbitals appears to be significantly below that of Jupiter or Neptune. The object is placed and removed from its horseshoe orbit by the mechanism of the precession of the nodes. This precession is accelerated by the perturbative effects of Saturn. The chaotic nature of the orbit of this object constrains the degree of predictability of its dynamical evolution on timescales longer than a few $10 \mathrm{kyr}$. This strongly suggests that its dynamical age is much shorter than that of the solar system; therefore, (83982) Crantor is unlikely to be a member of a hypothetical primordial population of objects moving in a $1: 1$ mean motion resonance with Uranus. Horner \& Evans (2006) claimed that Uranus cannot currently efficiently trap objects in the 1:1 commensurability even for short periods of time. Our results suggest that, contrary to this view and in spite of the destabilizing role of Saturn, Uranus still can actively capture temporary co-orbitals. Regarding the question of stability, (83982) Crantor's orbital inclination is close to the edge of one of the stability islands in $i$ identified by Dvorak et al. (2010), but $2010 \mathrm{EU}_{65}$ moves outside the stability islands proposed in that study, yet it seems to be more stable than (83982) Crantor. This 
comparatively better stability strongly suggests that not only inclination but also eccentricity play an important role in the longterm dynamics of these objects. In summary, key questions still remain open and more work is necessary to better understand the complex question of the stability of Uranian co-orbitals.

Acknowledgements. The authors thank the referee for his/her helpful suggestions regarding the presentation of this paper. The authors would like to thank S. J. Aarseth for providing the code used in this research. This work was partially supported by the Spanish "Comunidad de Madrid" under grant CAM S2009/ESP-1496 (Dinámica Estelar y Sistemas Planetarios). We thank M. J. Fernández-Figueroa, M. Rego Fernández and the Department of Astrophysics of Universidad Complutense de Madrid (UCM) for providing excellent computing facilities. Most of the calculations and part of the data analysis were completed on the "Servidor Central de Cálculo" of the UCM and we thank S. Cano Alsúa for his help during that stage. In preparation of this paper, we made use of the NASA Astrophysics Data System and the ASTRO-PH e-print server.

\section{References}

Aarseth, S. J. 2003, Gravitational N-Body Simulations (Cambridge: Cambridge University Press), 27

Alvarez-Candal, A., Barucci, M. A., Merlin, F., Guilbert, A., \& de Bergh, C. 2007, A\&A, 475, 369

Alvarez-Candal, A., Fornasier, S., Barucci, M. A., de Bergh, C., \& Merlin, F. 2008, A\&A, 487, 741

Bailey, B. L., \& Malhotra, R. 2009, Icarus, 203, 155

Barkume, K. M., Brown, M. E., \& Schaller, E. L. 2008, AJ, 135, 55

Brasser, R., Innanen, K. A., Connors, M., et al. 2004, Icarus, 171, 102

Brown, E. W. 1911, MNRAS, 71, 438

Christou, A. A., \& Asher, D. J. 2011, MNRAS, 414, 2965

Connors, M., Veillet, C., Brasser, R., et al. 2004, Meteorit. Planet. Sci., 39, 1251

Connors, M., Stacey, G., Brasser, R., \& Wiegert P. 2005, Plan. Space Sci., 53, 617

Cruikshank, D. P., Barucci, M. A., Emery, J. P., et al. 2007, in Protostars \& Planets V, eds. B. Reipurth, D. Jewitt, \& K. Keil (Tucson: University of Arizona Press), 879

Darwin, G. 1912, MNRAS, 72, 642

DeMeo, F. E., Fornasier, S., Barucci, M. A., et al. 2009, A\&A, 493, 283

Dermott, S. F., \& Murray, C. D. 1981a, Icarus, 48, 1

Dermott, S. F., \& Murray, C. D. 1981b, Icarus, 48, 12

Doressoundiram, A., Barucci, M. A., Tozzi, G. P., et al. 2005, Plan. Space Sci., 53,1501

Doressoundiram, A., Peixinho, N., Moullet, A., et al. 2007, ApJ, 134, 2186

Dvorak, R., Schwarz, R., Süli, Á., \& Kotoulas, T. 2007, MNRAS, 382, 1324

Dvorak, R., Bazsó, Á, \& Zhou, L.-Y. 2010, Celest. Mech. Dyn. Astron., 107, 51

Fraser, W. C., \& Brown, M. E. 2012, ApJ, 749, 33 de la Fuente Marcos, C., \& de la Fuente Marcos, R. 2012a, A\&A, 547, L2 de la Fuente Marcos, C., \& de la Fuente Marcos, R. 2012b, A\&A, 545, L9 de la Fuente Marcos, C., \& de la Fuente Marcos, R. 2012c, MNRAS, 427, 728 de la Fuente Marcos, C., \& de la Fuente Marcos, R. 2012d, MNRAS, 427, L85 Gallardo, T. 2006, Icarus, 184, 29

Garfinkel, B. 1977, AJ, 82, 368

Giacaglia, G. E. O. 1970, in Periodic Orbits, Stability and Resonances (Dordrecht: Reidel), 515

Gilmore, A. C., Pravec, P., Helin, E. F., et al. 2002, MPEC 2002-H03

Giorgini, J. D., Yeomans, D. K., Chamberlin, A. B., et al. 1996, BAAS, 28, 1158

Holman, M. J., \& Wisdom, J. 1993, AJ, 2015, 1987

Horner, J., \& Evans, N. 2006, MNRAS 367, L20

Horner, J., Evans, N. W., \& Bailey, M. E. 2004, MNRAS 354, 798

Karlsson, O. 2004, A\&A, 413, 1153

Ketchum, J. A., Adams, F. C., \& Bloch, A. M. 2013, ApJ, 762, 71

Makino, J. 1991, ApJ, 369, 200

Marzari, F., Tricarico, P., \& Scholl, H. 2003, A\&A, 410, 725

Michel, P., Froeschlé, C., \& Farinella, P. 1996, A\&A, 313, 993

Mikkola, S., Innanen, K., Wiegert, P., Connors, M., \& Brasser, R. 2006, MNRAS, 369,15

Milani, A., Carpino, M., Hahn, G., \& Nobili, A. M. 1989, Icarus, 78, 212

Murray, C. D., \& Dermott, S. F. 1999, Solar System Dynamics (Cambridge: Cambridge University Press), 97

Namouni, F. 1999, Icarus, 137, 293

Nesvorný, D., \& Dones, L. 2002, Icarus, 160, 271

Ortiz, J. L., Gutiérrez, P. J., Casanova, V., \& Sota, A. 2003, A\&A, 407, 1149

Rabe, E. 1961, AJ, 66, 500

Rabinowitz, D., Tourtellotte, S., \& Marsden, B. G. 2010, MPEC 2010-H80

Rabinowitz, D., Schwamb, M. E., Hadjiyska, E., \& Tourtellotte, S. 2012, AJ, 144,140

Smith, B. A., Reitsema, H. J., Fountain, J. W., \& Larson, S. M. 1980, BAAS, 12, 727

Standish, E. M. 1998, JPL Planetary and Lunar Ephemerides, DE405/LE405, Interoffice Memo. 312.F-98-048, Jet Propulsion Laboratory, Pasadena, California

Stansberry, J., Grundy, W., Brown, M., et al. 2008, in The Solar System Beyond Neptune, eds. M. A. Barucci, H. Boehnhardt, D. P. Cruikshank, \& A. Morbidelli (Tucson: University of Arizona Press), 161

Synnott, S. P., Peters, C. F., Smith, B. A., \& Morabito, L. A. 1981, Science, 212, 191

Tegler, S. C., Romanishin, W., \& Consolmagno, G. J. 2003, ApJ, 599, L49

Thuring, B. 1959, AN, 285, 71

Ticha, J., Tichy, M., Haver, R., et al. 2002, MPEC 2002-L55

Wajer, P., \& Królikowska, M. 2012, Acta Astron., 62, 113

Weissman, P. R., \& Wetherill, G. W. 1974, AJ, 79, 404

Wiegert, P., Innanen, K. A., \& Mikkola, S. 1997, Nature, 387, 685

Wiegert, P. A., Innanen, K. A., \& Mikkola, S. 1998, AJ, 115, 2604

Wiegert, P., Innanen, K., \& Mikkola, S. 2000, AJ, 119, 1978 